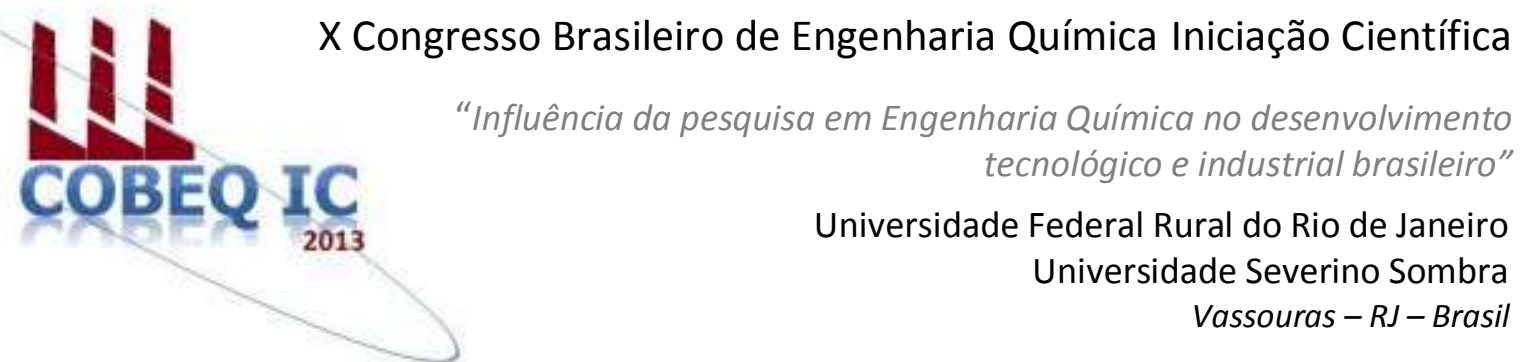

\title{
ANÁLISE DO DIÂMETRO DO ORIFÍCIO DE UNDERFLOW EM UM HIDROCICLONE FILTRANTE ÓTIMO (HFOT 1)
}

\author{
MARTINS*11 M. P. B.; SILVA $^{2}$, N. K. G. BARROZO ${ }^{3}$, M. A. S. \\ ${ }^{1}$ Aluno da FEQUI/UFU ${ }^{2}$ Mestranda da FEQUI/UFU ${ }^{3}$ Professor da FEQUI/UFU \\ Faculdade de Engenharia Química - Universidade Federal de Uberlândia \\ Endereço - UFU, Avenida João Naves de Ávila, 2121, Bloco K, Uberlândia, CEP. 38408-100, MG, \\ email: masbarrozo@ufu.br
}

\begin{abstract}
RESUMO - Hidrociclones são equipamentos que realizam a separação de misturas sólido-líquido, líquido-líquido e líquido-gás. Na forma convencional, esse separador consiste em uma seção cônica acoplada a uma seção cilíndrica, apresentando uma corrente de alimentação e duas correntes de saída (underflow e overflow). Propostas tem sido feitas para modificar essa estrutura convencional do hidrociclone, por exemplo, a incorporação de uma parede filtrante na região cônica (patente de pesquisadores da FEQUI/UFU) e a consequente vazão adicional de filtrado, que provou fornecer melhorias no desempenho do separador para determinadas condições experimentais. Uma vez que a configuração geométrica desse equipamento influencia significativamente no processo de separação, o objetivo deste trabalho foi estudar e quantificar, experimentalmente, o efeito do diâmetro do orifício de underflow na separação de um hidrociclone filtrante com geometria ótima (HFOT 1), obtido pelo uso conjunto das técnicas de Evolução Diferencial e superfícies de respostas, que fornece máxima eficiência de separação. De acordo com os principais resultados, observou-se redução na razão de líquido ao diminuir o diâmetro do orifício de underflow, este efeito foi quantificado.
\end{abstract}

Palavras chave: eficiência total de separação, sedimentação centrífuga, separação sólido-líquido.

\section{INTRODUÇÃO}

Hidrociclones são separadores sólidolíquido, líquido-líquido e líquido-gás. Devido à facilidade de construção e menor necessidade de espaço físico para sua instalação, se comparado a outros separadores, esses equipamentos tem tido destaque nos processos de separação.
O princípio de separação desses separadores é a sedimentação centrífuga, ou seja, as partículas suspensas são submetidas a uma aceleração centrífuga que as separa do fluido. Essa aceleração ocorre devido à entrada tangencial da corrente de alimentação na parte superior do cilindro, que induz o fluido a realizar, ao longo de sua trajetória, um movimento rotacional. A alimentação escoa de forma descendente dentro do hidrociclone e à 
medida que adentra na seção cônica as partículas mais densas são conduzidas ao orifício de underflow situado no vértice inferior do cone. Por outro lado, a parte fluida que contém as partículas mais finas, que não são descarregadas no underflow, migra para o centro do eixo do equipamento, formando um vórtice interno ascendente e com movimento rotacional inverso àquele criado pelo primeiro vórtice, e deixa o equipamento pelo orifício de overflow, localizado axialmente na parte superior do cilindro (Svarovsky, 1984).

Sabe-se que a geometria de um hidrociclone tem efeito decisivo no seu desempenho bem como no movimento das partículas no interior do equipamento (Petty e Parks, 2001). Dessa forma, têm sido propostas algumas alterações geométricas, para esses separadores, objetivando a melhoria nos seus desempenhos (Wang e Yu, 2006; Vieira et al., 2011).

Neste panorama, pesquisadores da Faculdade de Engenharia Química da Universidade Federal de Uberlândia (FEQUI/UFU) propuseram a incorporação de um cone permeável a um hidrociclone de geometria convencional, denominado hidrociclone filtrante, que por sua vez foi objeto de patente da referida instituição. $\mathrm{O}$ princípio de funcionamento do hidrociclone filtrante é análogo ao do equipamento convencional, acrescido do fato de que além das vazões volumétricas de alimentação, underflow e overflow, há uma corrente adicional de fluido, proveniente da coleta de filtrado produzido na superfície porosa do cone.

Tendo em vista a busca por melhorias no poder de classificação em hidrociclones filtrantes, em um estudo de otimização realizado na FEQUI/UFU mediante aplicação de planejamento de experimentos (Box et al., 1978) e superfícies de resposta, Vieira (2006) estudou 25 configurações geométricas de hidrociclones e obteve equações para previsão de alguns índices de desempenho (número de Euler, eficiência total de separação, diâmetro de corte e razão de líquido), em função de algumas variáveis geométricas: o diâmetro da alimentação $(D i)$, o diâmetro de overflow $(D o)$, o comprimento total do hidrociclone $(L)$ e o ângulo do tronco de cone $(\theta)$, sendo as três primeiras postas em termo de uma relação geométrica com o diâmetro da parte cilíndrica do hidrociclone $(D c)$.

A determinação da melhor configuração de operação caracteriza um problema de otimização. Tradicionalmente, a resolução deste problema tem sido obtida através do uso de métodos determinísticos, isto é, que fazem uso de informações do gradiente da função objetivo e das restrições para a atualização do projeto inicial adotado, sendo que sua utilização se dá devido a recursos computacionais. No entanto, o uso de algoritmos não determinísticos, que não fazem uso de informações do gradiente da função objetivo, tem atraído a atenção da comunidade científica, pois eles não investem todo o esforço computacional em torno de um único projeto inicial, são de fácil concepção conceitual e implementação e são facilmente estruturados em arquitetura paralela (Lobato, 2008).

O algoritmo de Evolução Diferencial (ED), proposto por Price e Storn (1997), configura-se como um interessante método não determinístico devido à qualidade dos resultados apresentados na literatura em diferentes aplicações na ciência e na engenharia.

Sabendo-se que a modificação na dimensão do diâmetro do orifício de underflow é capaz de alterar o poder de classificação de partículas em um hidrociclone, o objetivo deste trabalho foi avaliar o efeito do diâmetro do orifício de underflow sobre o poder de classificação do hidrociclone filtrante ótimo encontrado por meio da aplicação da técnica de Evolução Diferencial aliada a técnica de superfície de resposta e capaz de fornecer máxima eficiência de separação.

\section{MATERIAIS E MÉTODOS}

\section{Planejamento Experimental}

O algoritmo de Evolução Diferencial (ED) é capaz de trabalhar com variáveis de projeto discretas e contínuas. Foi utilizada uma otimização discreta com base no Planejamento Composto Central (PCC) de Vieira (2006), mostrado na Tabela 1, e objetivou-se a mudança no ângulo do tronco de cone $(\theta)$ bem como as razões, em relação ao diâmetro da 
parte cilíndrica, do diâmetro de alimentação $(D i / D c)$, do diâmetro de overflow $(D o / D c)$, e do comprimento total do hidrociclone $(L / D c)$. A técnica de ED foi responsável, então, por fazer todas as combinações possíveis entre essas quatro variáveis, de forma a fornecer uma geometria de hidrociclone que leva à máxima eficiência total, com base na equação empírica obtida por Vieira (2006) para esta resposta de interesse.

Tabela 1 - PCC para 4 fatores e 5 réplicas no centro (Vieira, 2006)

\begin{tabular}{|c|c|c|c|c|}
\hline Hidrociclone & $\begin{array}{c}D i / D c \\
\left(X_{1}\right)\end{array}$ & $\begin{array}{c}D o / D c \\
\left(X_{2}\right)\end{array}$ & $\begin{array}{c}L / D c \\
\left(X_{3}\right)\end{array}$ & $\begin{array}{c}\boldsymbol{\theta} \\
\left(\mathbf{X}_{4}\right)\end{array}$ \\
\hline H1 & 0,16 & 0,22 & 4,7 & $11,2^{\circ}$ \\
\hline H2 & 0,16 & 0,22 & 4,7 & $17,8^{\circ}$ \\
\hline H3 & 0,16 & 0,22 & 6,9 & $11,2^{\circ}$ \\
\hline H4 & 0,16 & 0,22 & 6,9 & $17,8^{\circ}$ \\
\hline H5 & 0,16 & 0,32 & 4,7 & $11,2^{\circ}$ \\
\hline H6 & 0,16 & 0,32 & 4,7 & $17,8^{\circ}$ \\
\hline H7 & 0,16 & 0,32 & 6,9 & $11,2^{\circ}$ \\
\hline H8 & 0,16 & 0,32 & 6,9 & $17,8^{\circ}$ \\
\hline H9 & 0,26 & 0,22 & 4,7 & $11,2^{\circ}$ \\
\hline H10 & 0,26 & 0,22 & 4,7 & $17,8^{\circ}$ \\
\hline H11 & 0,26 & 0,22 & 6,9 & $11,2^{\circ}$ \\
\hline H12 & 0,26 & 0,22 & 6,9 & $17,8^{\circ}$ \\
\hline H13 & 0,26 & 0,32 & 4,7 & $11,2^{\circ}$ \\
\hline H14 & 0,26 & 0,32 & 4,7 & $17,8^{\circ}$ \\
\hline H15 & 0,26 & 0,32 & 6,9 & $11,2^{\circ}$ \\
\hline H16 & 0,26 & 0,32 & 6,9 & $17,8^{\circ}$ \\
\hline H17 & 0,13 & 0,27 & 5,8 & $14,5^{\circ}$ \\
\hline H18 & 0,29 & 0,27 & 5,8 & $14,5^{\circ}$ \\
\hline H19 & 0,21 & 0,19 & 5,8 & $14,5^{\circ}$ \\
\hline H20 & 0,21 & 0,35 & 5,8 & $14,5^{\circ}$ \\
\hline H21 & 0,21 & 0,27 & 3,9 & $14,5^{\circ}$ \\
\hline H22 & 0,21 & 0,27 & 7,6 & $14,5^{\circ}$ \\
\hline H23 & 0,21 & 0,27 & 5,8 & $9^{\circ}$ \\
\hline H24 & 0,21 & 0,27 & 5,8 & $20^{\circ}$ \\
\hline H25(5) & 0,21 & 0,27 & 5,8 & $14,5^{\circ}$ \\
\hline
\end{tabular}

\section{Material Particulado}

O material particulado utilizado para a realização dos experimentos foi a rocha fosfática, fornecida pela Copebrás de Catalão/GO, cuja densidade real determinada pela técnica de picnometria a Hélio, foi equivalente a $3,237 \pm 0,006 \mathrm{~g} / \mathrm{cm}^{3}$.

\section{Unidade Experimental}

A unidade experimental de separação sólido-líquido em hidrociclones que esta montada no laboratório de Sistemas Particulados da FEQUI/UFU, conforme ilustra a Figura 1, é formada por um tanque com capacidade de 250 litros, responsável pelo armazenamento da suspensão. A fim de manter homogênea a concentração da suspensão durante a operação, existe um sistema de homogeneização formado por um agitador mecânico e um par de chicanas. Acoplada ao tanque de suspensão existe uma bomba helicoidal resistente à abrasão. Além disso, imediatamente anterior à entrada da alimentação no hidrociclone, está instalado um manômetro de Bourdon digital.

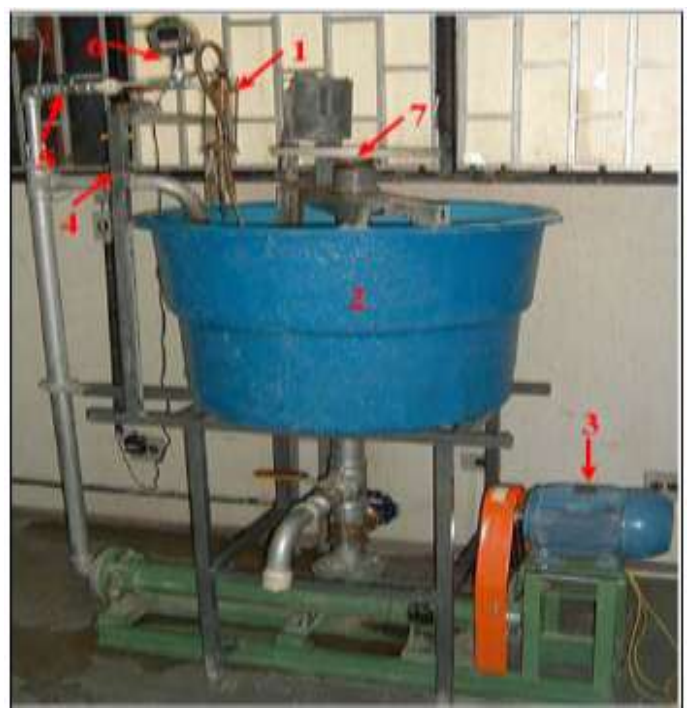

Figura 1 - Unidade experimental: (1) Hidrociclone; (2) Reservatório; (3) Bomba helicoidal; (4) Válvula de bypass; (5) Válvula; (6) Manômetro; (7) Agitador mecânico (Silva, 2012)

\section{Medidas e Condições Operacionais}

Os experimentos foram realizados para um valor fixo de queda de pressão: $-\Delta \mathrm{P}=1,47$ bar, para três valores de diâmetro do orifício de underflow ( $5 \mathrm{~mm}, 4,5 \mathrm{~mm}$ e $2,5 \mathrm{~mm}$ ) e com concentração de sólido no tanque de, aproximadamente, $1 \%$ em volume.

As concentrações mássicas da alimentação, underflow e overflow foram obtidas através de técnicas de gravimetria através de amostras coletadas e secas em estufa por volta de $24 \mathrm{~h}$ à $105^{\circ} \mathrm{C}$. As vazões volumétricas das correntes supracitadas também foram obtidas coletando uma quantidade de material de cada uma delas, cronometrando-se o tempo de coleta e 
pesando-se a amostra, possibilitando o cálculo da vazão mássica e, com a informação da densidade, da vazão volumétrica.

No estudo, a vazão volumétrica da alimentação foi calculada pela Equação 1.

$$
Q_{a}=\frac{W_{a}}{\rho_{a}}
$$

onde: $Q_{a}$ é vazão volumétrica da alimentação, $W_{a}$ a vazão mássica da alimentação e $\rho_{a}$ representa densidade da alimentação (fluido mais sólido).

De posse dos resultados obtidos para as vazões mássicas e concentrações mássicas das correntes de alimentação e underflow, calculou-se a eficiência total de separação a partir da Equação 2.

$$
\eta=\frac{C_{w u} \cdot W_{u}}{C_{w} \cdot W_{a}}
$$

O número adimensional de Euler $(\mathrm{Eu})$, que expressa a relação entre uma queda de pressão local (determinada pelo manômetro digital instalado na entrada do hidrociclone) e a energia cinética por unidade de volume na alimentação é obtido pela Equação 3.

$$
E u=\frac{\pi D_{C}^{4}(-\Delta P)}{8 \rho Q_{a}^{2}}
$$

A razão de líquido, quociente entre a vazão volumétrica de líquido do underflow e a vazão volumétrica de líquido da alimentação, é calculada pela Equação 4.

$$
R_{L}=\frac{Q_{u}\left(1-C_{v u}\right)}{Q_{a}\left(1-C_{v}\right)}
$$

para: $Q_{u}$ (vazão volumétrica de underflow), $C_{v}$ (concentração volumétrica de alimentação) e $C_{v u}$ (concentração volumétrica de underflow).

\section{Hidrociclone utilizado}

A geometria encontrada por meio das técnicas de otimização através do uso de superfície de resposta e ED, foi denominada HFOT1 (Hidrociclone Filtrante Otimizado 1), e está ilustrada na Figura 2. As dimensões reais do hidrociclone otimizado foram: $D i=$ $3,9 \mathrm{~mm}, D o=5,7 \mathrm{~mm}, L=174 \mathrm{~mm}, \theta=9^{\circ}$ e $\ell$ $=12 \mathrm{~mm}$.

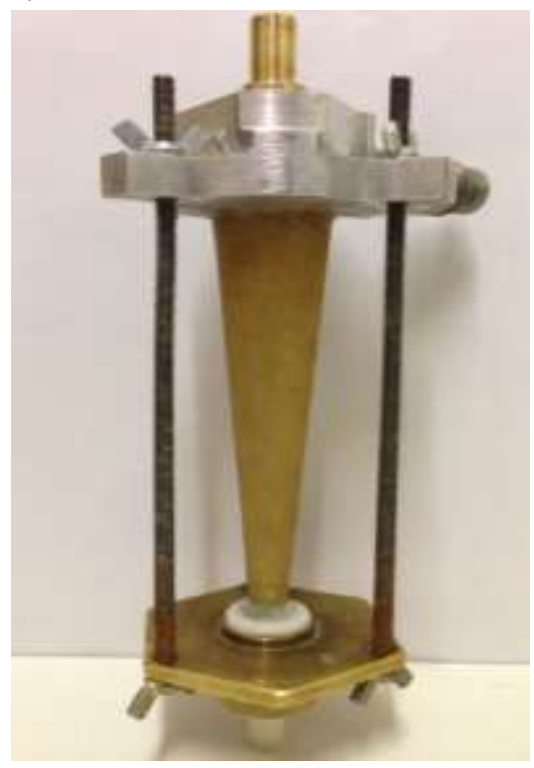

Figura 2 - HFOT1

\section{RESULTADOS E DISCUSSÕES}

Os resultados obtidos experimentalmente para o HFOT1, com a incorporação dos três diâmetros de orifício de underflow para uma queda de pressão fixa de 1,47 bar, foram analisados com base em algumas variáveis associadas ao desempenho de hidrociclone: vazão volumétrica da alimentação, número adimensional de Euler, razão de líquido e eficiência total de separação.

Com base na Figura 3, é possível observar que para o diâmetro do orifício de underflow igual a $5 \mathrm{~mm}$ foi encontrado o maior valor de vazão volumétrica da alimentação. É válido ressaltar que em hidrociclones, a vazão volumétrica da alimentação está relacionada com o gasto energético do equipamento, expresso em termos do número adimensional de Euler, ou seja, quanto maior a vazão de alimentação menor será o consumo energético $(\mathrm{Eu})$, isso pode ser observado pela analisando-se simultaneamente as Figuras 3 e 4. Embora o valor da vazão volumétrica de alimentação para $D u=5 \mathrm{~mm}$ tenha sido superior às demais vazões correspondentes aos outros valores de $D u$ (2,5 e 4,5 mm), observou-se que a influência de $D u$ na vazão de alimentação é pequena. 
A Figura 4 apresenta os valores obtidos para o número de Euler. Quando maior o valor de $D u$, menor foi o consumo energético apresentado pelo equipamento.

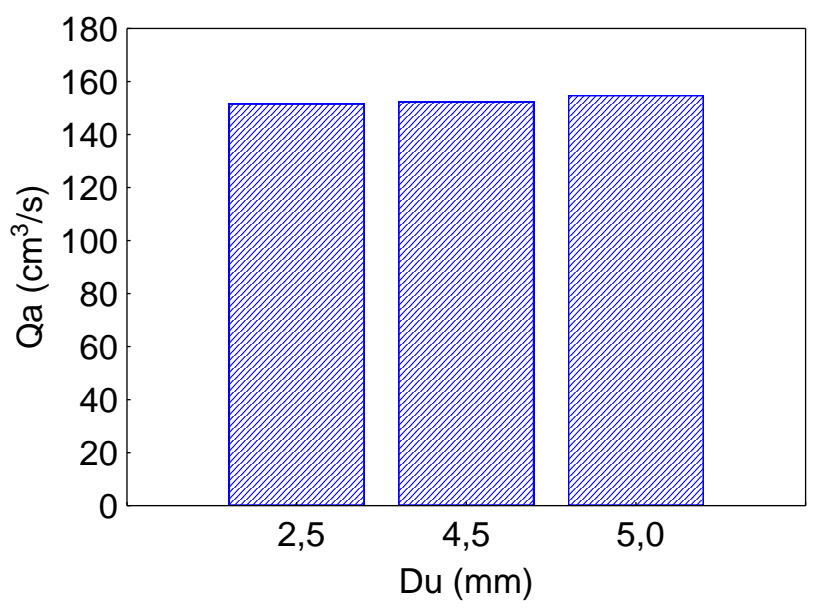

Figura 3 - Vazão de alimentação para $D u$ igual a $2,5,4,5$ e $5 \mathrm{~mm}$

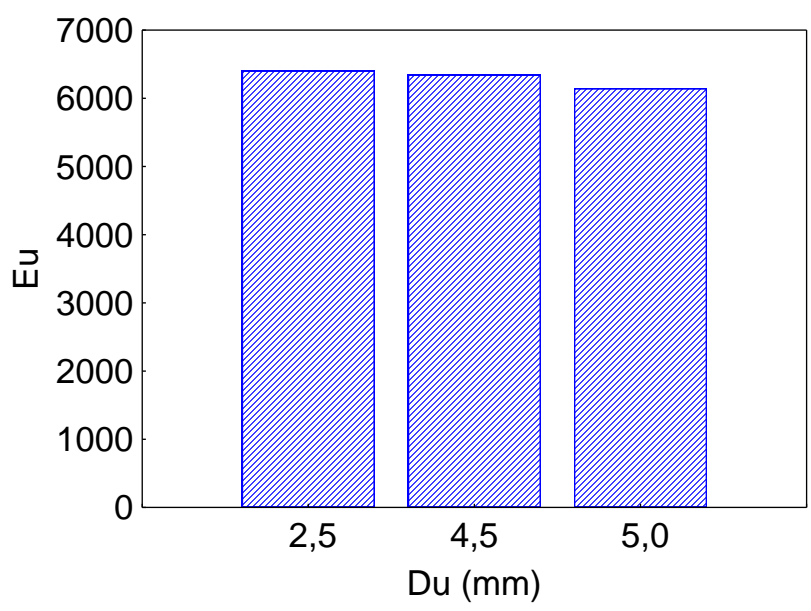

Figura 4 - Número de Euler para $D u$ igual a $2,5,4,5$ e $5 \mathrm{~mm}$

Quanto maior o $D u$, maiores foram os valores da razão de líquido, conforme ilustra a Figura 5. Para o diâmetro do orifício de underflow igual a $5 \mathrm{~mm}$ observa-se um salto no valor da razão líquido, se comparado ao menor $D u(2,5 \mathrm{~mm})$, e ainda um acréscimo de aproximadamente $10 \%$ em comparação ao valor obtido com a incorporação do diâmetro de underflow de $4,5 \mathrm{~mm}$. Uma vez que o diâmetro do orifício de underflow está diretamente relacionado com a razão de líquido (quanto maior o $D u$ maior $\mathrm{R}_{\mathrm{L}}$ ) é válido ressaltar que para o $D u$ de $5 \mathrm{~mm}$ a corrente de underflow apresenta maior porcentagem de líquido, dessa forma, é possível classificá-la como uma corrente de underflow mais diluída, ou menos concentrada em sólidos.

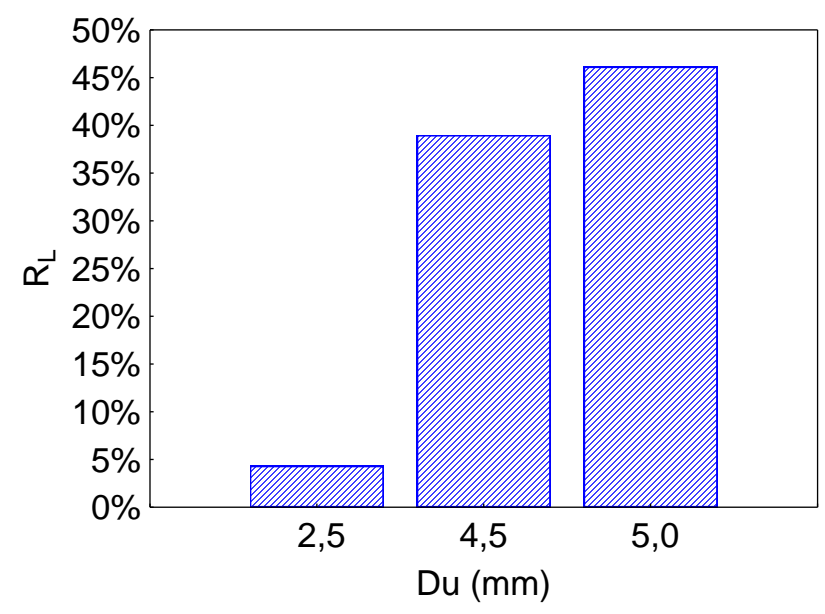

Figura 5 - Razão de liquido para Du igual a $2,5,4,5$ e 5 mm

A Figura 6 mostra os valores de eficiência total obtidos para o HFOT1 frente à variação do diâmetro de underflow (Du). Observa-se que quanto maior o $D u$, maior é o valor da eficiência. Isso é explicado pela análise conjunta das Figuras 5 e 6: quanto mais líquido é descarregado no underflow, mais partículas são arrastadas com ele e maior é a eficiência. Logo, para razões de líquido maiores, tem-se maiores eficiências.

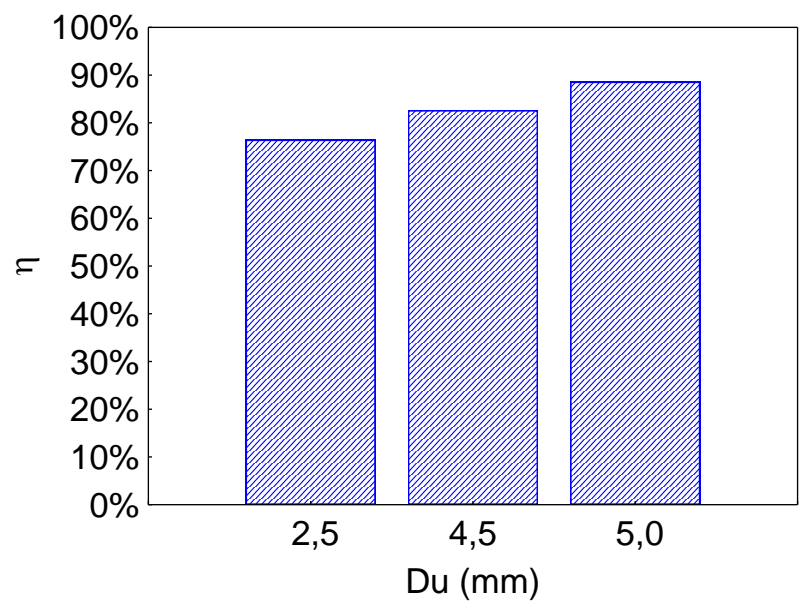

Figura 6 - Eficiência total para $D u$ igual a $2,5,4,5$ e 5 mm

\section{CONCLUSÃO}

Partindo-se de uma geometria de hidrociclone filtrante otimizada pelo uso conjunto das técnicas de Evolução Diferencial 
e de superfície de resposta, que fornecesse máxima eficiência total de separação, buscouse avaliar a influência do diâmetro do orifício de underflow no poder de classificação desse equipamento.

Os resultados mostraram que o melhor $D u$ a ser incorporado nesse separador é o de 5 $\mathrm{mm}$, pois para este caso, o equipamento apresentou alta eficiência de separação bem como baixo número de Euler, ou seja, menor consumo energético, quando comparados aos resultados obtidos para os demais diâmetros do orifício de underflow estudados.

\section{NOMENCLATURA}

$C_{w}$ - concentração volumétrica da alimentação $(\%)$

$C_{w u}$ - concentração mássica do underflow (\%)

$D c$ - diâmetro do cilindro do hidrociclone (mm)

$D u$ - diâmetro do orifício de underflow (mm)

$E u$ - número de Euler

HFOT1 - hidrociclone filtrante otimizado 1

$-\Delta \mathrm{P}$ - queda de pressão entre a saída da corrente de overflow e a corrente de alimentação do hidrociclone ( $\mathrm{Pa})$

$Q_{a}$ - vazão volumétrica da alimentação $\left(\mathrm{m}^{3} / \mathrm{s}\right)$

$\mathrm{R}_{\mathrm{L}}$ - razão de líquido (\%)

$W_{u}$ - vazão mássica do underflow $(\mathrm{g} / \mathrm{s})$

$\eta$ - eficiência total de separação (\%)

$\rho$ - densidade do fluido $\left(\mathrm{kg} / \mathrm{m}^{3}\right)$

\section{REFERÊNCIAS}

BOX, M. J.; HUNTER, W. G.; HUNTER, J. S. (1978), Statistics for Experimenters An Introduction to Design, Data Analysis and Model Building, J. Wiley and Sons, New York-EUA

LOBATO, F.S., (2008), Otimização Multiobjetivo para o Projeto de Sistemas de Engenharia. UFU, Uberlândia - MG, (Tese de Doutorado) 402p.

PETTY, C. A., PARKS S. M., (2001), "Flow predictions within hydrocyclones". Filtration and Separation, v. 38, p. 28-34.

PRICE, K., STORN, R. (1997), "Differential evolution - a simple evolution strategy for fast optimization". Dr. Dobb's Journal, v.22, p.18-24.
SILVA, D.O., (2012), Otimização da Separação Sólido-Líquido em Hidrociclones Mediante Modificações Geométricas. PGEQ/Faculdade de Engenharia Química da UFU, Uberlândia - MG, (Tese de Doutorado) 189p

SVAROVSKY, L., (1984), Hydrocyclone, Holt, Rinehart \& Winston, Eastbourne, UK. 198p.

VIEIRA, L. G. M., (2006), Otimização dos Processos de Separação em Hidrociclones Filtrantes, PPGEQ/Faculdade de Engenharia Química da UFU, Uberlândia - MG, (Tese de Doutorado).

VIEIRA, L. G. M.; SILVÉRIO, B. C.; DAMASCENO, J. J. R.; BARROZO, M. A. S., (2011), "Performance of hydrocyclones with different geometries", The Canadian Journal of Chemical Engineering, v. 89, n. 4, p. 655-662.

WANG, B., YU, A. B., (2006). "Numerical study of particle-fluid flow in hydrocyclones with different body dimensions". Min. Eng., v. 19, p. 10221033

\section{AGRADECIMENTOS}

Os autores agradecem à CAPES e ao CNPq pelo subsídio financeiro que possibilitou a realização deste trabalho. 